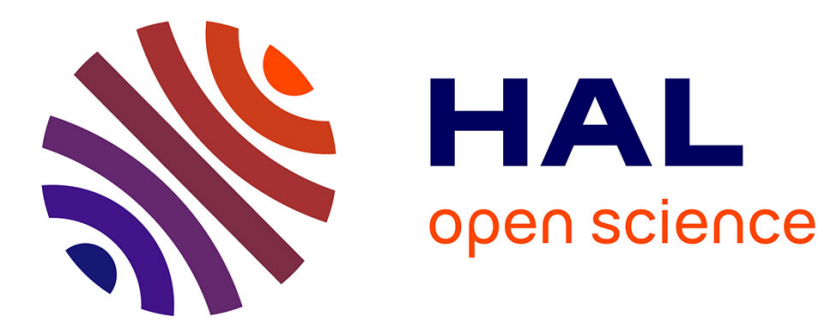

\title{
Two-Stage Adaptive Designs In Nonlinear Mixed Effects Models: Application To Pharmacokinetics In Children
}

Cyrielle Dumont, Marylore Chenel, France Mentré

\section{To cite this version:}

Cyrielle Dumont, Marylore Chenel, France Mentré. Two-Stage Adaptive Designs In Nonlinear Mixed Effects Models: Application To Pharmacokinetics In Children. 2014. inserm-01076940

\section{HAL Id: inserm-01076940 https://www.hal.inserm.fr/inserm-01076940}

Preprint submitted on 23 Oct 2014

HAL is a multi-disciplinary open access archive for the deposit and dissemination of scientific research documents, whether they are published or not. The documents may come from teaching and research institutions in France or abroad, or from public or private research centers.
L'archive ouverte pluridisciplinaire HAL, est destinée au dépôt et à la diffusion de documents scientifiques de niveau recherche, publiés ou non, émanant des établissements d'enseignement et de recherche français ou étrangers, des laboratoires publics ou privés. 
TWO-STAGE ADAPTIVE DESIGNS IN NONLINEAR MIXED EFFECTS MODELS: APPLICATION TO PHARMACOKINETICS IN CHILDREN

Cyrielle Dumont ${ }^{1}$, Marylore Chenel ${ }^{2}$, and France Mentré ${ }^{1}$

${ }^{1}$ IAME, UMR 1137, INSERM, F-75018 Paris, France; Univ Paris Diderot, Sorbonne Paris Cité, F-75018 Paris, France.

${ }^{2}$ Division of Clinical Pharmacokinetics, Institut de Recherches Internationales Servier, Suresnes, France

cyrielle.dumont@inserm.fr

Key Words: Adaptive design, design optimization, nonlinear mixed effects models, Population pharmacokinetics. 


\section{INTRODUCTION}

In clinical pharmacokinetics (PK), the evolution of drug concentration versus time is studied. PK profiles are described by mathematical models, and nonlinear mixed effects models (NLMEM) (Sheiner et al., 1972 ; Sheiner and Steimer, 2000) are used to analyze PK data obtained in group of patients or healthy volunteers. This is called the population approach. PK studies in children are often more difficult to perform than those in adults. The blood volume that can be taken in children is much more limited than in adult healthy volunteers so that the number of sampling times is limited. For ethical reasons, PK studies in children are conducted in patients who may potentially benefit from the treatment, and not in healthy volunteers, as for adults. As, most of the time, only sparse data can be obtained in children, NLMEM is an appropriate methodology for analyzing PK information in pediatric trials both from a practical and ethical point of view (EMA, 2006 ; Mentré et al., 2001 ; Tod et al., 2008).

The design of PK studies is important when the number of samples and subjects is limited, as every sample must be informative, especially when clinical constraints are strong. Indeed, the design has a large impact on the precision of population parameter estimates (Mentré et al., 1997). A PK design for NLMEM consists in choosing the number of patients and for each patient the elementary design. Elementary designs are composed of several sampling times to be drawn for each individual. In the EMA guideline related to PK studies in the pediatric population (EMA, 2006), simulations or theoretical optimal design approaches, based on prior knowledge, are presented as tools to be considered for the selection of sampling times, number of subjects and number of samples per subject. To avoid simulations, which are time consuming, designs can be evaluated using the Fisher information matrix $\left(M_{F}\right)$ and the optimization of its determinant, corresponding to the D-optimality criterion. The calculation of $M_{F}$ for NLMEM was first developed by Mentré et al. (1997) and Retout et al. (2002) for uniresponse NLMEM and then extended to multiresponse population PK/pharmacodynamic (PD) models (Bazzoli et al., 2009 ; Gueorguieva et al., 2007 ; Retout et al., 2002) using a first-order Taylor expansion of the population PK model around the random effect (Mentré et al., 1997 ; Retout et al., 2001). Several approximations of $M_{F}$ can be used in the community of PKPD design, but it was suggested (Mielke and Schwabe, 2010 ; Mielke, 2012) that the block diagonal expression might be more reliable than one with the full $M_{F}$, when an approach by linearization is performed. The calculation of the $M_{F}$ for NLMEM used in population PK is implemented in several software packages, including PFIM developed in $\mathrm{R}$, dedicated to design evaluation and optimization (Bazzoli et al., 2010). PFIM evaluates and optimizes population designs in NLMEM with single and multiple responses (Bazzoli et 
al., 2009). The expression of $M_{F}$ in NLMEM was also extended to evaluate and to optimize designs in crossover trials (Nguyen et al., 2012) and is implemented into the version PFIM 3.2 , available since 2010. To evaluate $M_{F}$ in NLMEM, prior information is needed such as the model but also a priori values of the parameters. This is called a local design (Burstein et al., 1997). Local design based on these a priori values can lead to a sub-optimal design when the true population parameter values are different from the a priori ones. Alternatives to local design are robust design, relying on a priori distribution of parameters (Dodds et al., 2005 ; Foo et al., 2012 ; Pronzato and Walter, 1988), or adaptive design (Chow and Chang, 2007).

Adaptive design is increasingly used for randomized clinical trials or for dose-ranging studies (Dragalin et al., 2010 ; EMA, 2009 ; FDA, 2006 ; FDA, 2010). The working group Pharmaceutical Research and Manufacturers of America (PhRMA, Dragalin, 2006) defined an adaptive design as a design of experiment in which accumulated data during the trial are used to possibly modify the aspects of the study, without compromising the validity and integrity of the clinical trial (Gallo et al., 2006).

It was already shown that adaptive designs improve dose ranging during clinical drug development (Bornkamp et al, 2007). Adaptive designs are now widely developed for randomized clinical trials or dose-ranging studies, but are barely used in population PK/PD (Foo and Duffull, 2012 ; Zamuner et al., 2010). However, according to a survey performed by Mentré et al. (2013), adaptive design in population PK/PD is a priority for pharmaceutical companies, graduating its importance with a median equal to 4 on a scale between 0 and 5 . Zamuner et al. (2010) showed that optimal adaptive design provided a more efficient design for the study they conducted in imaging. Indeed, the optimal adaptive design allowed to minimize the number of subjects needed for the study and to maximize information. Foo and Duffull (2012) developed and evaluated a method allowing to conduct PK adaptive bridging studies. Using simulation studies, the authors showed that D-optimal adaptive method applied to bridging studies was more efficient, since it provided better estimations, when the PK profiles of the target and a priori populations differed.

An important point in adaptive design is the number of adaptations, that is cohorts of patients. In a study concerning the dose-ranging optimal design, it was shown that twostage adaptive designs are more efficient than fully adaptive designs, i.e., when adaptation is performed after each patient (Fedorov et al., 2012). In the framework of a clinical trial in cancerology, Chen (1997) compared three-stage adaptive designs to two-stage adaptive designs. Comparison showed that the benefit of a three-stage adaptive design compared to a two-stage design is not as important as the gain of two-stage design versus one stage. They also discussed that an adaptive design with four stages or more would not allow to improve 
the results. Furthermore, two-stage adaptive designs are easier to conduct in clinical trials as only one modification is made.

The first objective of the present work was to introduce two-stage adaptive design in NLMEM. In section 2, we present the model and the principle of the two-stage adaptive design, and the development of $M_{F}$ associated to this design is described in Section 3 . The second objective was to evaluate, with a simulation approach, the impact of two-stage designs on the precision of parameter estimation when the true PK parameters are different from the a priori ones. We have performed this simulation for a population PK study in paediatrics. And finally, we have investigated the influence of the sample size ratio between the two stages of an adaptive design. Section 4 presents the simulation study and the results are given in Section 5 . 


\section{Model and design}

A NLMEM, or a population model, is defined as follows. The elementary design $\xi_{i}$ of individual $i(i=1, \ldots, N)$, is defined by $n_{i}$ sampling times and their allocation in time, that is $\xi_{i}=\left(t_{i 1}, \ldots, t_{i n_{i}}\right)$. The vector of observations $Y_{i}$ for the $i^{t h}$ individual is defined as

$$
Y_{i}=f\left(g\left(\beta, b_{i}\right), \xi_{i}\right)+\varepsilon_{i}
$$

with

$$
g\left(\beta, b_{i}\right)=\beta+b_{i}
$$

for a normal distribution of parameters (additive random effects), or

$$
g\left(\beta, b_{i}\right)=\beta \exp \left(b_{i}\right)
$$

for a lognormal distribution of parameters (exponential random effects). $\beta$ represents the pvector of the fixed effects parameters and $b_{i}$, the vector of the $p$ random effects for individual $i$. It is assumed that $b_{i} \sim N(0, \Omega)$ with $\Omega$ defined as a $p \times p$ diagonal matrix, for which, each diagonal element $\omega_{r}^{2}, r=1, \ldots, p$, represents the variance of the $r^{\text {th }}$ component of the vector $b_{i}$. The function $f$ defines the nonlinear structural model. $\varepsilon_{i}$ is the vector of residual error and it is also supposed that $\varepsilon_{i} \sim N\left(0, \Sigma_{i}\right)$ with $\Sigma_{i}$ a $n_{i} \times n_{i}$-diagonal matrix such that:

$$
\Sigma_{i}\left(\beta, b_{i}, \sigma_{\text {inter }}, \sigma_{\text {slope }}, \xi_{i}\right)=\operatorname{diag}\left(\sigma_{\text {inter }}+\sigma_{\text {slope }} \times f\left(g\left(\beta, b_{i}\right), \xi_{i}\right)\right)^{2} .
$$

The parameters $\sigma_{\text {inter }}$ and $\sigma_{\text {slope }}$ are respectively the additive and proportional parts of the error model. The case $\sigma_{\text {slope }}=0$ corresponds to a homoscedastic error model, whereas the case $\sigma_{\text {inter }}=0$ corresponds to a constant coefficient of variation error model. The case where the two parameters differ from zero is called a combined error model. Finally, conditionnally on the value of $b_{i}$, it is assumed that the errors $\varepsilon_{i}$ are independently distributed. Let $\lambda^{T}=\left(\omega_{1}^{2}, \ldots, \omega_{p}^{2}, \sigma_{\text {inter }}, \sigma_{\text {slope }}\right)$ be the vector of variance terms and let $\Psi$ be the vector of the population parameters to be estimated, so that $\Psi^{T}=\left(\beta^{T}, \lambda^{T}\right)$.

A one-stage population design for NLMEM is composed of $N$ individuals to whom an elementary design $\xi_{i}, i=1, \ldots, N$, is allocated. A population design is therefore described by $N$ elementary designs for a total number $n_{t o t}$ of observations such as $n_{t o t}=\sum_{i=1}^{N} n_{i}$ and $\Xi=\left\{\xi_{1}, \ldots, \xi_{N}\right\}$. In the case where the elementary design $\xi$ is the same for the $N$ individuals, the total number of observations is $n_{t o t}=N \times n$. 
A two-stage population design is composed of 2 groups of $N_{1}$ and $N_{2}$ individuals $\left(N_{1}+\right.$ $\left.N_{2}=N\right)$ with respectively designs $\Xi_{1}$ and $\Xi_{2}$ within each group. When the design is assumed identical within each group, the population design can then be written as follows: $\Xi=\left\{\left[\xi_{1}, N_{1}\right] ;\left[\xi_{2}, N_{2}\right]\right\}$. In a two-stage adaptive design, $\Xi_{1}$ is determined from a priori parameters $\Psi_{0}$ and $\Xi_{2}$ is optimized for the parameters $\hat{\Psi}_{1}$ estimated with the data of the first cohort. More precisely, at the first stage, from the model and a priori parameters $\Psi_{0}$, data are collected for $N_{1}$ individuals according to a design $\Xi_{1}$, optimized using the a priori parameters. From data $Y_{1}$, obtained with the first cohort, the population parameters $\hat{\Psi}_{1}$ are estimated. At the second stage, the design $\Xi_{2}$ of the second cohort, composed of $N_{2}$ individuals, is then optimized for the parameters $\hat{\Psi}_{1}$, estimated at the end of the first stage. Data $Y_{2}$ are then collected for the $N_{2}$ individuals having the design $\Xi_{2}$ and finally, data $Y_{1}$ and $Y_{2}$, obtained from $N=N_{1}+N_{2}$ individuals included in each cohort, are simultaneously analyzed to estimate parameters $\hat{\Psi}_{2}$.

Here, we use the D-optimality criterion to optimize designs which consists in maximizing the determinant of the Fisher information matrix $\left(\operatorname{det}\left(M_{F}\right)\right)$. We suppose that all individuals have the same elementary design $\xi$ for a same stage; that is $\xi_{1}$ corresponds to $\Xi_{1}$ and $\xi_{2}$ to $\Xi_{2}$. The sizes of cohorts $N_{1}$ and $N_{2}$ are fixed, as well as the number of samples $n_{1}$ and $n_{2}$ within each elementary design. Therefore, we only optimize with respect to the sampling times in $\xi_{1}$ and $\xi_{2}$. Figure 1 represents a two-stage adaptive design.

\section{Fisher information matrix}

In the following, the index $i$ for the individual is omitted for sake of simplicity. The elementary Fisher information matrix $M_{F}(\Psi, \xi)$ for an individual with design $\xi$ is given by

$$
M_{F}(\Psi, \xi)=E\left(-\frac{\partial^{2} L(\Psi ; Y)}{\partial \Psi \partial \Psi^{T}}\right)
$$

where $L(\Psi ; Y)$ is the log-likelihood of the vector of observations $Y$ of that individual for the population parameters $\Psi$. Because $f$ is nonlinear, there is no analytical expression for the loglikelihood and a first-order Taylor expansion (Mentré et al., 1997) of the model $f(g(\beta, b), \xi)$, around the expectation of $b$, that is to say around 0 , is used. Using this linearization, the statistical model can be written as:

$$
Y \cong f(g(\beta, 0), \xi)+\left(\frac{\partial f^{T}(g(\beta, b), \xi)}{\partial b}\right)_{b=0} b+\varepsilon
$$


The approximated marginal expectation $E$ and variance $V$ of $Y$ are given by:

$$
\begin{gathered}
E(Y) \cong E=f(g(\beta, 0), \xi) \\
\operatorname{Var}(Y) \cong V=\left(\frac{\partial f^{T}(g(\beta, b), \xi)}{\partial b}\right)_{b=0} \Omega\left(\frac{\partial f(g(\beta, b), \xi)}{\partial b^{T}}\right)_{b=0}+\Sigma\left(\beta, 0, \sigma_{\text {inter }}, \sigma_{\text {slope }}, \xi\right)
\end{gathered}
$$

The log-likelihood $L$ is approximated by:

$$
-2 L(\Psi ; Y) \cong n \ln (2 \pi)+\ln (|V|)+(Y-E)^{T} V^{-1}(Y-E) .
$$

For a linear mixed effects model, this tacitly assumes that the random variable $Y$ is approximately Gaussian. Hence for NLMEM the approximation is better when the level of non-linearity of the model is limited and/or when inter-individual variability is small.

The elementary $M_{F}$ depends on the approximated marginal expectation $E$ and variance $V$ of the observations. Assuming that the derivative of $V$ does or does not depend on the fixed effects, the elementary $M_{F}$ is a full matrix or a block diagonal matrix. There is no clear consensus on what is the best approximation, but here in our approach by linearization, we assume the choice of the block diagonal expression (Mielke and Schwabe, 2010 ; Nyberg et $a l ., 2014)$. With that approximation, we have:

$$
M_{F}(\Psi, \xi) \cong \frac{1}{2}\left(\begin{array}{cc}
A(E, V) & 0 \\
0 & B(E, V)
\end{array}\right)
$$

where

$$
(A(E, V))_{m l}=2 \frac{\partial f^{T}}{\partial \beta_{m}} V^{-1} \frac{\partial f}{\partial \beta_{l}}
$$

with $m$ and $l=1, \ldots, p$ and

$$
(B(E, V))_{m l}=\operatorname{tr}\left(\frac{\partial V}{\partial \lambda_{m}} V^{-1} \frac{\partial V}{\partial \lambda_{l}} V^{-1}\right)
$$

with $m$ and $l=1, \ldots, \operatorname{dim}(\lambda)$.

In the case of only one group composed of $N$ individuals, the population Fisher information matrix for a population design $\Xi$ is computed as:

$$
M_{F}(\Psi, \Xi)=N \times M_{F}(\Psi, \xi)
$$


This calculation of $M_{F}$ is implemented in PFIM (Bazzoli et al., 2010).

When neglecting the dependence implied by the adaptation in the representation of the Fisher matrix, the two-stage design is performed as follows. For the two-stage adaptive design, we assume $N_{1}$ individuals associated with a same design $\xi_{1}$ at the first stage, and $N_{2}$ individuals associated with a same design $\xi_{2}$ at the second stage. For the first stage of a two-stage adaptive design, the Fisher information matrix is expressed as follows:

$$
M_{F}\left(\Psi_{0}, N_{1} \xi\right)=N_{1} M_{F}\left(\Psi_{0}, \xi\right)
$$

We define $\xi_{1}$ as the design which maximizes the determinant of this matrix. Then for the second stage, the expression of $M_{F}$ is:

$$
M_{F}\left(\hat{\Psi}_{1}, N_{1} \xi_{1}+N_{2} \xi\right)=N_{1} M_{F}\left(\hat{\Psi}_{1}, \xi_{1}\right)+N_{2} M_{F}\left(\hat{\Psi}_{1}, \xi\right)
$$

For the second stage, $\xi_{2}$ is the design which maximizes the determinant of this matrix, using the parameters $\hat{\Psi}_{1}$ estimated at the first stage. This expression is implemented in version 4.0 of PFIM which will be soon available.

\section{SIMULATION STUDY}

\subsection{Pharmacokinetic example}

The simulation study mimicked a pediatric PK trial. The PK model is a two-compartment model with first-order absorption, exponential random effects and proportional error model. The observed concentration of a child at sampling time $t_{j}$ is modelled by:

$$
f\left(\theta, t_{j}\right)=D\left(A e^{-\alpha \cdot t_{j}}+B e^{-\beta \cdot t_{j}}-(A+B) e^{-k a . t_{j}}\right)
$$

where $\theta=(k a, C L, V 1, Q, V 2)$ and

$$
\begin{gathered}
\beta=\frac{1}{2}\left(\frac{Q}{V 1}+\frac{Q}{V 2}+\frac{C L}{V 1}-\sqrt{\left.\left(\frac{Q}{V 1}+\frac{Q}{V 2}+\frac{C L}{V 1}\right)^{2}-4 \frac{Q}{V 2} \frac{C L}{V 1}\right)},\right. \\
\alpha=\frac{\frac{Q}{V 2} \frac{C L}{\beta 1}}{\beta}, \\
A=\frac{k a}{V 1} \frac{\frac{Q}{V 2}-\alpha}{(k a-\alpha)(\beta-\alpha)}, \\
B=\frac{k a}{V 1} \frac{\frac{Q}{V 2}-\beta}{(k a-\beta)(\alpha-\beta)} .
\end{gathered}
$$


In equation (16), $D$ denotes the dose, $k a$ the absorption rate constant, $C L$ the clearance of elimination of the drug, $Q$ the inter-compartmental clearance from compartment 1 to compartment 2, $V 1$ and $V 2$ the volumes respectively of the central compartment and of the peripheral compartment. With the chosen parametrization, note that the parameters vary independently of each other.

Here we assumed that the dose administered is equal to $0.1 \mathrm{mg}$. We defined two sets of pharmacokinetic parameters: the a priori parameters $\Psi_{0}$, guessed from adults, and the true parameters $\Psi^{*}$ with which all simulations are performed. They are presented in Table 1. All parameters have the same variance $\left(\omega^{2}=0.3\right)$ and we consider the same proportional error $(\sigma=0.2)$.

The optimal designs with one or two stages are obtained assuming a total number $N=60$ children, having the same design $\xi$ at a same stage composed of 5 sampling times among 15 possible sampling times: $0.083,0.17,0.25,0.33,0.5,0.75,1,2,3,4,5,6,8,10,12$ hours after drug administration. Designs are optimized with PFIM (version 3.2), which allows us to maximize the determinant of $M_{F}$ using statistical design within a finite set of sampling times (Retout et al., 2007). Designs are then evaluated through the criterion given by $\operatorname{det}\left(M_{F}\right)^{1 / P}$, $P$ being the total number of parameters in $\Psi$. We denote by $\xi_{1}$ and $\xi^{*}$ the designs optimized respectively with the parameters $\Psi_{0}$ and $\Psi^{*}$, and by $\xi_{2}$ the design optimized at the second stage. Optimal designs obtained with the parameters $\Psi_{0}$ and $\Psi^{*}$ are respectively $\xi_{1}=0.083,1,2,5,12$ hours after drug administration and $\xi^{*}=0.083,0.33,0.75,2,12$ after drug administration. Figure 2 represents mean profiles obtained with the parameters $\Psi_{0}$ and $\Psi^{*}$, on which are added the times of $\xi_{1}$ and $\xi^{*}$.

\subsection{Simulation settings}

We consider designs with a total number of $N=60$ children. All simulated data are obtained with the true parameters $\Psi^{*}$. We are interested in 2 one-stage designs (non adaptive designs), that is the designs $\xi_{1}$ (60-0), optimized with parameters $\Psi_{0}$, and the optimal design $\xi^{*}(0-60)$, optimized with the true parameters (Figure 2). In parallel, we are interested in 3 two-stage adaptive designs: $\xi_{50-10}(50-10), \xi_{30-30}(30-30), \xi_{10-50}(10-50)$, varying the size of each cohort $N_{1}$ and $N_{2}$ : 50 and 10, 30 and 30, 10 and 50 children, at the first stage and at the second stage, respectively.

For each of the designs mentioned above, we simulate 100 adaptive clinical trials with $\mathrm{R}$. The simulation study is performed as follows. From the same design $\xi_{1}$, we simulate 10 data sets $\left(Y_{1}^{1}, \ldots, Y_{1}^{10}\right)$ for the first cohort composed of $N_{1}$ children with the parameters $\Psi^{*}$. From 
these 10 data sets, 10 vectors of parameters $\hat{\Psi}_{1}$ are estimated using saemix (http://cran.rproject.org/web/packages/saemix/index.html) in R. The saemix package implements the Stochastic Approximation Expectation Maximisation (SAEM) algorithm (Comets et al., 2011), which computes the maximum likelihood estimator of the population parameters in NLMEM, without any approximation of the model. Then, for each vector of parameters $\hat{\Psi}_{1}$, a design $\xi_{2}$ is optimized with the combined Fisher information matrix described by equation (15). 10 simulations are then performed for each of the 10 designs $\xi_{2}$ for the $N_{2}$ children of the second cohort. Thus, we obtain 100 data sets $\left(Y_{2}^{1,1}, \ldots, Y_{2}^{10,10}\right)$, and we combine data $Y_{1}$ and $Y_{2}$ from which we estimate 100 vectors of parameters $\hat{\Psi}_{2}$ using saemix. The simulation scheme of adaptive clinical trials is described in Figure 3.

\subsection{Evaluation methods}

Our aims are to evaluate the impact of two-stage adaptive designs on the precision of parameter estimation on the one hand, and to investigate the influence of the sample size ratio of each stage on the other hand. To evaluate a design, we compute the relative root mean square errors (RRMSE) for the 100 estimated $\hat{\Psi}_{2}$ of the 2 one-stage designs, $\xi_{1}$ and $\xi^{*}$, and of the 3 adaptive two-stage designs, $\xi_{50-10}, \xi_{30-30}$, and $\xi_{10-50}$, as follows:

$$
\operatorname{RRMSE}\left(\Psi_{p}\right)=\frac{\sqrt{E\left(\left(\hat{\Psi}_{2 p}-\Psi_{p}^{*}\right)^{2}\right)} \times 100}{\Psi_{p}^{*}}
$$

with $\hat{\Psi}_{2 p}$ the estimated p component and $\Psi_{p}^{*}$ the true value. We also calculate the standardized RRMSE for each parameter and each design as the RRMSE divided by the RRMSE obtained with $\xi^{*}, \xi^{*}$ being the reference because it is the design optimized with the true parameters. For each design, mean standardized RRMSE is then computed.

We are also interested in the criterion predicted by PFIM, associated with the evaluation of one-stage designs. The criterion evaluates the amount of information and is computed with the true parameters $\Psi^{*}$. For the two-stage designs, we compute a mean criterion, defined as the mean of the 10 criterion values obtained for each of the 10 two-stage designs computed with $\Psi^{*}$. Indeed, the design $\xi_{1}$ is identical for all data sets at the first stage but we have 10 designs $\xi_{2}$ at the second stage, optimized according to the 10 data sets at the first stage.

It is of interest to note that we could have optimized the designs with respect to the RRMSE-criterion. However this is more time consuming as there is no expression of the expected RRMSE and hence the criterion has to be evaluated by simulation. This is why 
most approaches use the D-optimality criterion, which indeed assumes no bias and uses an approximation for the expected $M_{F}$.

In section 5.1 we present the comparison of the RRMSE and of the criteria for the onestage (non adaptive) designs, and for the two-stage design $\xi_{30-30}$, with the same number of children at the first and second stages. Then, we focus in Section 5.2 on the comparison of RRMSE and criteria of two-stage adaptive designs, with different sizes of cohort for the first and second stages ( $N_{1}$ and $N_{2}$ respectively equal to 50 and 10, 30 and 30, 10 and 50).

\section{RESULTS}

\subsection{Precision of parameter estimation}

For the one-stage designs, the RRMSE and standardized RRMSE of each parameter, computed from the 100 vectors of estimated parameters $\hat{\Psi}_{2}$ are presented in Table 2. As expected, we can see that the RRMSE are lower for the design $\xi^{*}$, which corresponds to the optimal design, and are high for the design $\xi_{1}$, optimized from a priori parameters $\Psi_{0}$. These results are summarized by the mean standardized RRMSE, which is equal to 1.76 for $\xi_{1}$, that is almost the double of the one for the optimal design $\xi^{*}$ which equals to 1 (reference value). Regarding the criterion for $\xi_{1}$, computed with $\Psi^{*}$, its value is 26.9 , much lower than the criterion value for $\xi^{*}$, equal to 84.7. It underlines the low information obtained with $\xi_{1}$.

For the two-stage design with $N_{1}=N_{2}=30$, the 10 optimal designs $\xi_{2}$ obtained for the 10 vectors of parameters $\hat{\Psi}_{1}$ are displayed in Figure 4 (notice that only 6 different designs are obtained). These designs $\xi_{2}$ are closer to $\xi^{*}$ than to $\xi_{1}$ and two of them coincide with $\xi^{*}$. The RRMSE for this two-stage design $\xi_{30-30}$, computed from the 100 vectors of parameters $\hat{\Psi}_{2}$ are close to the RRMSE obtained for the optimal design $\xi^{*}$ (see Table 2). For some parameters (for instance fixed effects CL and V2) the optimal design $\xi^{*}$ provides slightly greater RRMSE than the adaptive design, whereas it provides better RRMSE for other parameters and a better overall mean RRMSE. This is because a global optimization criterion on all parameters was used and also because the adaptive design gives very good results (mean standardized RRMSE of 1.06). Indeed, the mean standardized RRMSE of $\xi_{30-30}$ is equal to 1.06 and is therefore close to the value obtained for $\xi^{*}$ (equal to 1 ). The mean (respectively min and max) criterion values for the 10 two-stage design $\xi_{30-30}$, evaluated in $\Psi^{*}$, are 72.5 (respectively 69.1 and 74.6), which is close to the one for the optimal design. We can see that two-stage design has allowed a correction of the unsatisfactory results obtained for the non adaptive design $\xi_{1}$. 


\subsection{Impact of size of each cohort}

For the 3 two-stage designs with various number of children $\left(\xi_{50-10}, \xi_{30-30}\right.$ and $\left.\xi_{50-10}\right)$, the RRMSE and standardized RRMSE, computed from the 100 vectors of parameters $\hat{\Psi}_{2}$, are presented in Table 3. The RRMSE are slightly worse for the design $\xi_{10-50}$ when the number of children for the first cohort is low. Indeed, its associated mean standardized RRMSE is equal to 1.15 whereas the mean standardized RRMSE for the two-stage adaptive designs $\xi_{30-30}$ and $\xi_{50-10}$ are closer to the one for the optimal design and are respectively equal to 1.06 and 1.07. Nevertheless, this trend is not confirmed by the mean (respectively min and $\max$ ) criterion values since the later for the design $\xi_{10-50}$ equals 72.2 (respectively, 45.2 and $82.0)$ and is in the same range as the mean criterion obtained for the design $\xi_{30-30}$. The mean (respectively min and max) criterion values obtained for the design $\xi_{50-10}$ are 59.0 (respectively 56.5 and 60.9 ). The difference between the minimum criterion and the maximum criterion for the design $\xi_{10-50}$ is important, contrary to the design $\xi_{30-30}$. The minimum and maximum criterion values for the designs $\xi_{30-30}$ and $\xi_{50-10}$ are rather close, indicating that 30 subjects in the first cohort are enough to significantly reduce the variability of the design used at the second stage. Other investigations would be needed to confirm or infirm this observation. 


\section{DISCUSSION}

We have proposed an approach for two-stage design for NLMEM (with an implementation that will be available soon in the next version PFIM 4.0). In the present work, the calculation of $M_{F}$ was performed using a first-order linearization of the model by the Taylor expansion. The derivative of the variance of observations according to the fixed effects was assumed to be zero, which corresponds to a block diagonal expression for $M_{F}$. There is no clear consensus on what is the best approximation but it was suggested (Mielke and Schwabe, 2010 ; Mielke, 2012) that the block diagonal expression might be more reliable than one with the full $M_{F}$. In the simulation study on two examples (Nyberg et al., 2014), when using first order approximation, the block matrix gave better results. On the other hand, we did not take into account the adaptation in the optimization of the $M_{F}$ of the first step, nor that estimation was performed after first step for definition of $M_{F}$ of the second step. Hence, the dependence implied by the adaptation was neglected (Pronzato and Pazman, 2013; Fedorov and Leonov, 2013).

Using simulations, we have studied the impact of two-stage designs on the precision of parameter estimation for a population PK study in children. Saemix in R was used for parameter estimation and evaluation and optimization were performed with PFIM in R. We compared the results obtained for non adaptive designs, that is with the total number of children $N=60$ having the design $\xi_{1}$, optimized with the wrong parameters, and with the total number of children having the design $\xi^{*}$, optimal for the true parameters, to the results obtained for the two-stage adaptive design, with the same number of children at each of the two stages $\left(N_{1}=N_{2}=30\right)$. Results showed that the the non adaptive design $\xi_{1}$ provides poor precisions of parameter estimates. The adaptive two-stage design allows to compensate these poor results and yields performance close to those obtained for $\xi^{*}$, optimized with the true parameters.

Then, we investigated the influence of the ratio of sample size of each stage. For that, we studied two-stage designs with a number of children for each stage equal to 50/10, 30/30 or $10 / 50$, respectively. The RRMSE were sometimes less satisfactory for the third design whose the first cohort is small. The results obtained for two-stage adaptive designs were generally satisfactory for each of the three ratios considered and close to the ones obtained for the design $\xi^{*}$.

Other studies would be needed and it would also be important to increase the number of replications in the simulation study which is rather low. Indeed, we estimated 100 vectors of parameters at the end of the second stage but only 10 at the end of the first stage. The reason why we have limited the number of replications is the absence of automatic connec- 
tion between saemix and PFIM, although both are written in R. Consequently, a manual intervention at the end of the first stage is needed to incorporate into PFIM the vectors of parameters $\hat{\Psi}_{1}$ estimated with saemix and which are needed for the optimization of designs $\xi_{2}$. One perspective of this work would be to create an automatic connection to allow an important number of replications and for the use of this approach in clinical trials.

One limitation of this work was the assumptions made to conduct this simulation study. Indeed, we chose to fix the size of each cohort. Moreover, we imposed to have the same elementary design for all children in each cohort with a fixed number of sampling times. Although these choices represent constraints usually needed in clinical trial, other scenario should be studied. Also, further simulation studies are needed to set the balance between size of first and second cohorts and with other examples. One theoretical study could also be performed to determine the balance between the sizes of first and second cohorts in NLMEM, as already done by Pronzato and Pazman (2013) for generalized nonlinear models.

In this work, we have assumed that the model was known and correct. Indeed, the same a priori model at the beginning of the simulation study (for the first stage) was used for the second stage. No sensitivity analysis with respect to model misspecification was performed in the present work. Another step in adaptive design would be to use model averaging approaches (Claeskens and Lid Hjort, 2008).

In conclusion, local design, usually conducted for PK studies in children, relies on several strong hypotheses. Indeed, optimal design in NLMEM depends on the PK model and its parameters. Besides the structure, an a priori value should be given for each parameter of the model. We showed that the adaptive two-stage design is a promising approach for pediatric PK studies, relatively easy to conduct in clinical trials as there is only one interim analysis. In the example considered, two-stage adaptive designs provided satisfactory parameter estimates, close to the ones obtained for the optimal design with the true parameters ans thus allowed a correction of the poor results obtained for the non adaptive design optimized with the wrong a priori parameters. In conclusion, although adaptive designs are still barely used in population PK, results are promising and should stimulate the conduction of PK studies with this approach and help convince pharmaceutical companies of its interest (Mentré et al., 2013). 


\section{ACKNOWLEDGMENTS}

The research leading to these results has received support from the Innovative Medicines Initiative Joint Undertaking under grant agreement $\mathrm{n}^{\circ} 115156$, resources of which are composed of financial contributions from the European Union's Seventh Framework Programme (FP7/2007-2013) and EFPIA companies' in kind contribution. The DDMoRe project is also financially supported by contributions from Academic and SME partners. 


\section{REFERENCES}

Bandyopadhyay N, Dragalin V. Implementation of an adaptive group sequential design in a bioequivalence study. Pharmaceutical Statistics, 2007; 6: 115-22.

Bazzoli C, Retout S, Mentré F. Design evaluation and optimisation in multiple response non linear mixed effects models: PFIM 3.0. Computer Methods and Program in Biomedecine, 2010; 98: $55-65$.

Bazzoli C, Retout S, Mentré F. Fisher information matrix for nonlinear mixed effects multiple response models: evaluation and appropriateness of the first order linearization using a pharmacokinetic/pharmacodynamic model. Statistics in Medicine, 2009; 28: 1940-56.

Bornkamp B, Bretz F, Dmitrienko A, Enas G, Gaydos B, Hsu CH, König F, Krams M, Liu Q, Neuenschwander B, Parke T, Pinheiro J, Roy A, Sax R, Shen F. Innovative approaches for designing and analyzing adaptive dose-ranging trials. Journal of Biopharmaceutical Statistics, 2007; 17: 965-95.

Burstein AH, Gal P, Forrest A. Evaluation of a sparse sampling strategy for determining vancomycin pharmacokinetics in preterm neonates: application of optimal sampling theory. The Annals of Pharmacotherapy, 1997; 31: 980-3.

Chen TT. Optimal three-stage designs for phase II cancer clinical trials. Statistics in Medicine, 1997; 16: 2701-11.

Chow SC, Chang M. Adaptive design methods in clinical trials. Chapman and Hall/CRC; Boca Raton: 2007.

Claeskens G, Lid Hjort N. Model selection and model averaging. Cambridge Series in Statistical and Probabilistic Mathematics; Cambridge: 2008.

Comets E, Lavenu A, Lavielle M. SAEMIX, an R version of the SAEM algorithm, Population Approach Group in Europe, Athens, Greece, 2011;

Abstr 1695 [www . page-meeting.org/default.asp?abstract=2173].

Dodds MG, Hooker AC, Vicini P. Robust population pharmacokinetic experiment design. Journal of Pharmacokinetics and Pharmacodynamics, 2005; 32: 33-64.

Dragalin V. Adaptive designs: terminology and classification. Drug Information Journal, 2006; 40: 425-35. 
Dragalin V, Bornkamp B, Bretz F, Miller F, Padmanabhan S, Patel N, Perevozskaya I, Pinheiro J, Smith J. A simulation study to compare new adaptive dose-ranging designs. Statistics in Biopharmaceutical Research, 2010; 2: 487-512.

Dragalin V, Hsuan F, Padmanabhan SK. Adaptive designs for dose-finding studies based on sigmoid Emax model. Journal of Biopharmaceutical Statistics, 2007; 17: 1051-70.

European Medicines Agency. Guideline on the role of pharmacokinetics in the development of medicinal products in the paediatric population. http://www.ema.europa.eu/docs/en_ GB/document_library/Scientific_guideline/2009/09/WC500003066.pdf, 2006.

European Medicines Agency. Reflection paper on methodological issues in confirmatory clinical trials planned with an adaptive design. http://www.ema.europa.eu/docs/en_GB/document_ library/Scientific_guideline/2009/09/WC500003616.pdf, 2009.

Fedorov V, Leonov S. Optimal design for nonlinear response models. Chapman $\&$ Hall/CRC Biostatistics Series; Boca Raton: 2014.

Fedorov V, Wu Y, Zhang R. Optimal dose-finding designs with correlated continuous and discrete responses. Statistics in Medicine, 2012; 31: 217-34.

Food and Drug Administration. Critical Path Opportunities List. http://www.fda.gov/oc/initiatives/criticalpath/, 2006.

Food and Drug Administration. Guidance for Industry: adaptive design clinical trials for drugs and biologics.

http://www.fda.gov/downloads/drugs/guidancecomplianceregulatoryinformation/ guidances/ucm201790.pdf, 2010.

Foo LK, Duffull S. Adaptive optimal design for bridging studies with an application to population pharmacokinetic studies. Pharmaceutical Research, 2012; 29: 1530-43.

Foo LK, McGree J, Eccleston J, Duffull S. Comparison of robust criteria for D-optimal designs. Journal of Biopharmaceutical Statistics, 2012; 22: 1193-1205.

Gallo P, Chuang-Stein C, Dragalin V, Gaydos B, Krams M, Pinheiro J, PhRMA Working Group. Adaptive designs in clinical drug development - an executive summary of the PhRMA Working Group. Journal of Biopharmaceutical Statistics, 2006; 16: 275-83.

Gueorguieva I, Ogungbenro K, Graham G, Glatt S, Aarons L. A program for individual and population optimal design for univariate and multivariate response pharmacokinetic and 
pharmacodynamic models. Computer Methods and Programs in Biomedicine, 2007; 86: 5161.

Liu Q, Proschan MA, Pledger GW. A unified theory of two-stage adaptive designs. Journal of American Statistical Association, 2002; 97: 1034-41.

Mentré F, Chenel M, Comets E, Grevel J, Hooker A, Karlsson MO, Lavielle M, Gueorguieva I. Current use and developments needed for optimal design in pharmacometrics: a study performed amongst DDMoRe's EFPIA members. Clinical Pharmacology and Therapeutics: Pharmacometrics and Systems Pharmacology, 2013; 2: e46.

Mentré F, Dubruc C, Thénot J.P. Population pharmacokinetic analysis and optimization of the experimental design for Mizolastine solution in children. Journal of Pharmacokinetics and Pharmacodynamics, 2001; 28: 299-319.

Mentré F, Mallet A, Baccar D. Optimal design in random-effects regression models. Biometrika, 1997; 84: 429-442.

Mielke T. Approximations of the Fisher information for the construction of efficient experimental designs in nonlinear mixed effects models. Ph.D. thesis, Otto-von-Guericke- Universität Magdeburg, 2012.

Mielke T, Schwabe R. Some considerations on the Fisher information in nonlinear mixed effects models. Proceedings of the 9th international workshop in model-oriented design and analysis, Bertinoro, Italy, 2010.

Nguyen TT, Bazzoli C, Mentré F. Design evaluation and optimisation in crossover pharmacokinetic studies analysed by nonlinear mixed effects models. Statistics in Medecine, 2012; 31: 1043-1058.

Nyberg J, Bazzoli C, Ogungbenro K, Aliev A, Leonov S, Duffull S, Hooker A, Mentré F. Methods and software tools for design evaluation in population pharmacokinetics-pharmacodynamics. British Journal of Clinical Pharmacology, 2014; in press.

Pronzato L, Pazman A. Design of experiments in nonlinear models. Springer; New York: 2013.

Pronzato L, Walter E. Robust experiment design via maximin optimization. Mathematical Biosciences, 1988; 89: 161-76.

Retout S, Comets E, Samson A, Mentré F. Design in nonlinear mixed effects models: Optimization using the Fedorov-Wynn algorithm and power of the Wald test for binary covariates. 
Statistics in Medicine, 2007; 26: 5162-5179.

Retout S, Duffull S, Mentré F. Development and implementation of the population Fisher information matrix for the evaluation of population pharmacokinetic designs. Computer Methods and Programs in Biomedicine, 2001; 65: 141-51.

Retout S, Mentré F, Bruno R. Fisher information matrix for non-linear mixed-effects models: evaluation and application for optimal design of enoxaparin population pharmacokinetics. Statistics in Medicine, 2002; 21: 2623-39.

Saemix, http://cran.r-project.org/web/packages/saemix/index.html.

Sheiner LB, Rosenberg B, Melmon KL. Modelling of individual pharmacokinetics for computeraided drug dosage. Computers and Biomedical Research, 1972; 5: 411-459.

Sheiner LB, Steimer JL. Pharmacokinetic/pharmacodynamic modelling in drug development. Annual Review of Pharmacology and Toxicology, 2000; 40: 67-95.

Tod M, Jullien V, Pons G. Facilitation of drug evaluation in children by population methods and modelling. Clinical Pharmacokinetics, 2008; 47: 231-43.

Zamuner S, Di Iorio VL, Nyberg J, Gunn RN, Cunningham VJ, Gomeni R, Hooker AC. AdaptiveOptimal Design in PET occupancy Studies. Clinical Pharmacology and Therapeutics, 2010; 87: $563-71$. 
Table 1: Population pharmacokinetic parameter values

\begin{tabular}{|c|c|c|}
\hline Parameters & $\Psi_{0}$ & $\Psi^{*}$ \\
\hline ka $\left(h^{-1}\right)$ & 3.0 & 14 \\
CL $\left(L . h^{-1}\right)$ & 1.5 & 1.0 \\
V1 $(\mathrm{L})$ & 2.0 & 1.0 \\
Q $\left(L . h^{-1}\right)$ & 1.0 & 2.0 \\
V2 $(\mathrm{L})$ & 1.5 & 2.0 \\
\hline
\end{tabular}


Table 2: RRMSE computed for each parameter from the 100 vectors of parameters $\hat{\Psi}_{2}$ for each studied design

\begin{tabular}{|c|c|ccc|}
\cline { 3 - 5 } \multicolumn{2}{c|}{} & \multicolumn{3}{c|}{ RRMSE \% (standardized RRMSE) } \\
\hline Parameters & Values & $\xi_{1}(60-0)$ & $\xi_{30-30}(30-30)$ & $\xi^{*}(0-60)$ \\
\hline ka $\left(h^{-1}\right)$ & 14 & $\mathbf{1 6 0}(7.05)$ & $27.3(1.20)$ & 22.7 \\
CL $\left(L . h^{-1}\right)$ & 1 & $7.17(1.07)$ & $5.77(0.857)$ & 6.73 \\
V1 $(\mathrm{L})$ & 1 & $25.2(1.65)$ & $23.7(1.55)$ & 15.3 \\
Q $\left(L . h^{-1}\right)$ & 2 & $27.1(1.67)$ & $18.8(1.16)$ & 16.2 \\
V2 $(\mathrm{L})$ & 2 & $11.4(1.00)$ & $9.73(0.854)$ & 11.4 \\
$\omega_{k a}^{2}$ & 0.3 & $\mathbf{1 0 0}(1.21)$ & $\mathbf{9 0 . 4}(1.10)$ & $\mathbf{8 2 . 4}$ \\
$\omega_{C L}^{2}$ & 0.3 & $17.0(0.950)$ & $18.3(1.02)$ & 17.9 \\
$\omega_{V 1}^{2}$ & 0.3 & $48.0(1.33)$ & $38.3(1.06)$ & 36.0 \\
$\omega_{Q}^{2}$ & 0.3 & $\mathbf{8 9 . 9}(1.50)$ & $\mathbf{7 1 . 5}(1.19)$ & $\mathbf{5 9 . 9}$ \\
$\omega_{V 2}^{2}$ & 0.3 & $36.4(1.17)$ & $26.6(0.853)$ & 31.2 \\
$\sigma_{\text {slope }}$ & 0.2 & $10.4(0.765)$ & $10.8(0.794)$ & 13.6 \\
\hline Mean standardized & 1.76 & 1.06 & 1.00 \\
\multicolumn{2}{|c}{ RRMSE } & & & \\
\hline
\end{tabular}

${ }^{a}$ RRMSE in bold are RRMSE greater than $30 \%$ for the fixed effects and greater than $50 \%$ for the random effects. 
Table 3: RRMSE computed for each parameter from the 100 vectors of parameters $\hat{\Psi}_{2}$ for each studied design

\begin{tabular}{|c|c|ccc|}
\cline { 2 - 5 } \multicolumn{1}{c|}{} & \multicolumn{3}{c|}{ RRMSE \% (standardized RRMSE) } \\
\hline Parameters & Values & $\xi_{10-50}$ & $\xi_{30-30}$ & $\xi_{50-10}$ \\
\hline ka $\left(h^{-1}\right)$ & 14 & $\mathbf{3 0 . 3}(1.33)$ & $27.3(1.20)$ & $\mathbf{3 2 . 8}(1.44)$ \\
CL $\left(L . h^{-1}\right)$ & 1 & $7.07(1.05)$ & $5.77(0.857)$ & $4.88(0.725)$ \\
V1 $(\mathrm{L})$ & 1 & $19.3(1.26)$ & $23.7(1.55)$ & $21.1(1.38)$ \\
Q $\left(L . h^{-1}\right)$ & 2 & $21.2(1.31)$ & $18.8(1.16)$ & $19.2(1.19)$ \\
V2 $(\mathrm{L})$ & 2 & $12.3(1.08)$ & $9.73(0.854)$ & $11.4(1.00)$ \\
$\omega_{k a}^{2}$ & 0.3 & $\mathbf{8 6 . 3}(1.05)$ & $\mathbf{9 0 . 4}(1.10)$ & $\mathbf{8 2 . 6}(1.00)$ \\
$\omega_{C L}^{2}$ & 0.3 & $30.6(1.71)$ & $18.3(1.02)$ & $21.4(1.20)$ \\
$\omega_{V 1}^{2}$ & 0.3 & $23.5(0.653)$ & $38.3(1.06)$ & $37.1(1.03)$ \\
$\omega_{Q}^{2}$ & 0.3 & $\mathbf{7 8 . 2}(1.31)$ & $\mathbf{7 1 . 5}(1.19)$ & $\mathbf{6 8 . 0}(1.14)$ \\
$\omega_{V 2}^{2}$ & 0.3 & $34.1(1.09)$ & $26.6(0.853)$ & $34.3(1.10)$ \\
$\sigma_{\text {slope }}$ & 0.2 & $11.0(0.809)$ & $10.8(0.794)$ & $8.14(0.599)$ \\
\hline Mean standardized & 1.15 & 1.06 & 1.07 \\
\multicolumn{2}{|c|}{ RRMSE } & & & \\
\hline
\end{tabular}

${ }^{a}$ RRMSE in bold are RRMSE greater than $30 \%$ for the fixed effects and greater than $50 \%$ for the random effects. 


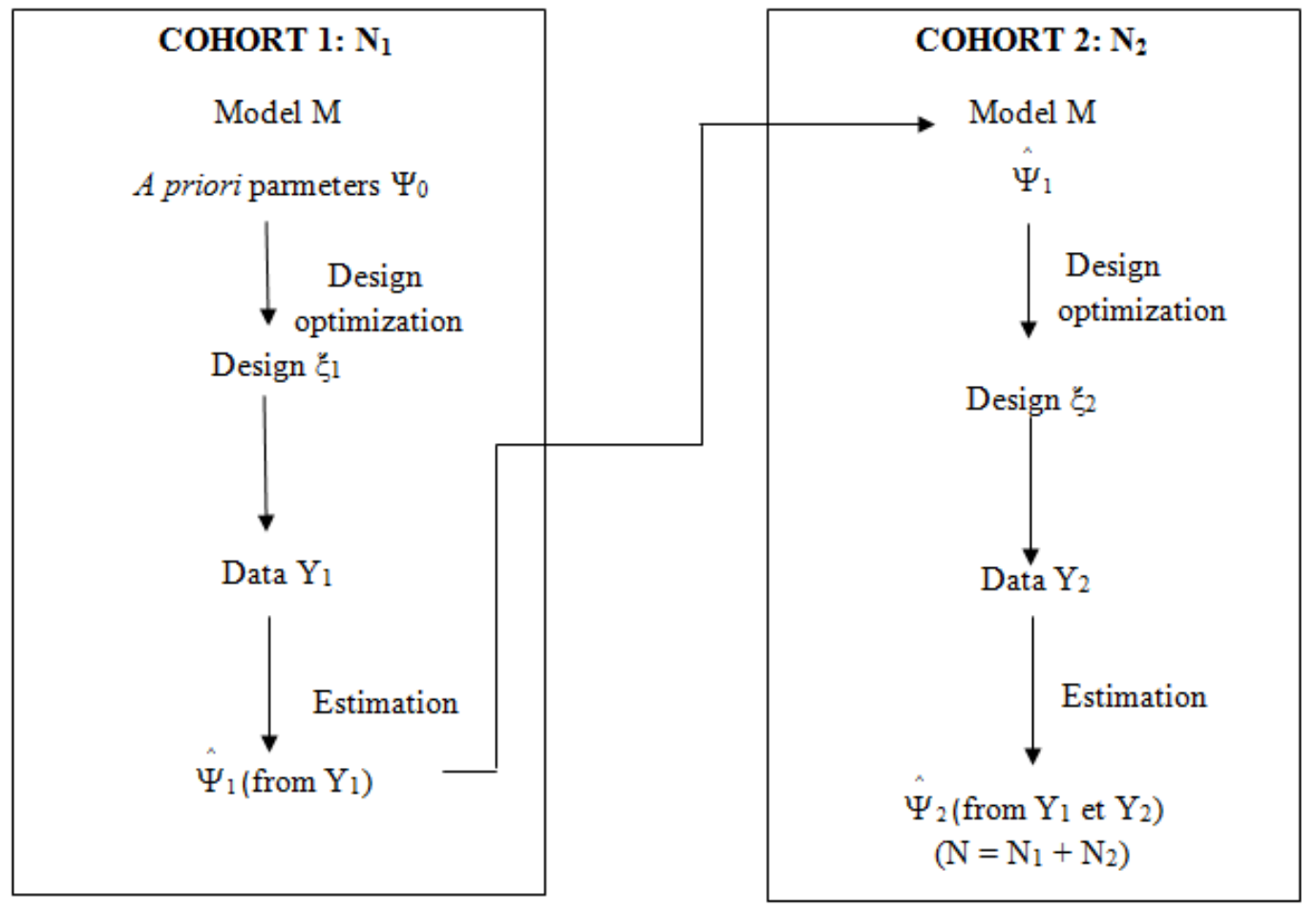

Figure 1: Schematic representation of a two-stage adaptive design. 


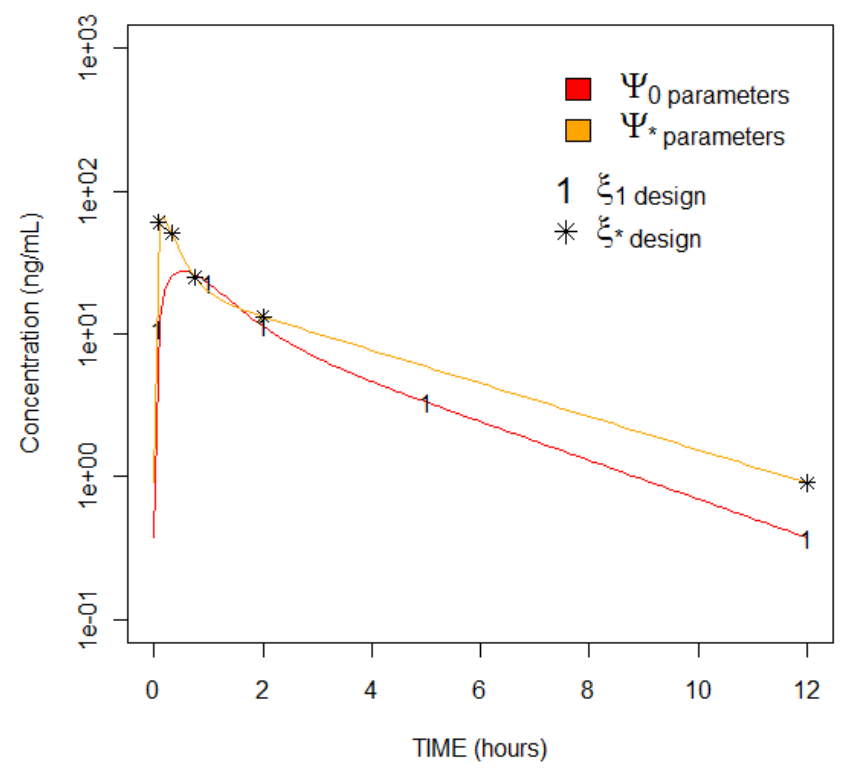

Figure 2: Mean PK profiles in semi-log scale for the 2 sets of parameters and associated optimal design with 5 samples. 


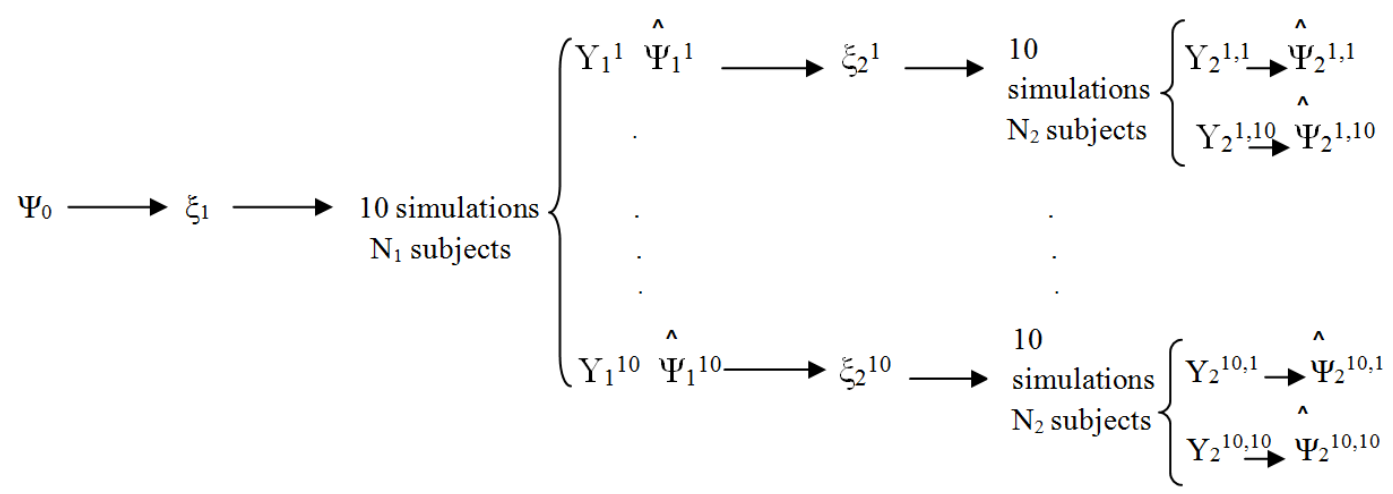

Figure 3: Simulation plan of 100 two-stage adaptive clinical trials. 


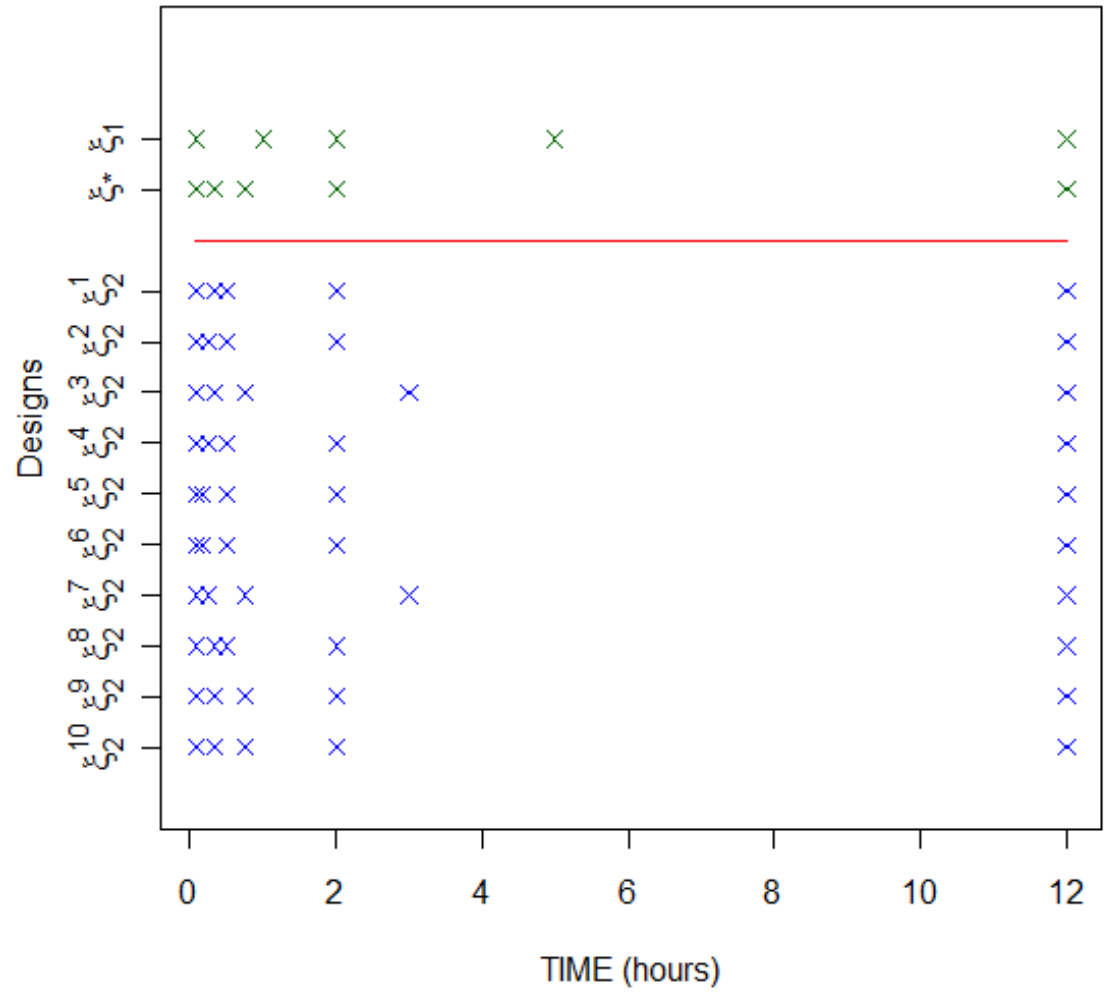

Figure 4: The 10 second-stage designs $\xi_{2}$ optimized from the 10 estimated $\hat{\Psi}_{1}$. 